\title{
About Relation between the Measure of Conflict and Decreasing of Ignorance in Theory of Evidence
}

\author{
Alexander Lepskiy ${ }^{1}$ \\ ${ }^{1}$ National Research University - Higher School of Economics, Moscow, Russia
}

\begin{abstract}
There is a problem of applying of combining rules to evidence which were got from different information sources in framework of Dempster-Shafer theory. In this work the conflict measure and index of decreasing of ignorance in frame of Dempster-Shafer theory are introduced for characterization of quality of applied combining rules. Those functionals are analyzed on the bodies of evidences of special type. It is shown that the great correlation between the bodies of evidence is a sufficient condition of decreasing of ignorance after the applying of combining rule. The relationships between the measure of conflict and the index of decreasing of ignorance in the case of different types of evidences and actions of various combining rules are investigated.
\end{abstract}

Keywords: Theory of evidence, combining rules, changing of ignorance, conflict measure.

\section{Introduction}

The combining rules are considered in Dempster-Shafer theory $[2,14]$ (theory of evidence, theory of belief function) for fusion of information that was obtained from various sources. The Dempster's rule was a first combining rule [2]. This rule has subjected to numerous critics [3, 4, 17-20]. As a result, new combining rules were suggested. All these rules have an advantages and disadvantages. They can give the correct result in one situation and non correct result in other situation. This related with following reasons: 1) availability of conflict of evidence; 2) availability the great deficiency of information in evidences (ignorance of evidence); 3 ) different interpretability of evidence. We won't analyze the third reason in this article but we will focus on first and second reasons.

The effectiveness of applying of combining rule may be estimated by quantity of decreasing of ignorance after the using of combining rule. The quantity of ignorance of evidence we will calculate with help of imprecise indices. Those indices were researched in many works in imprecise probability theory. In this article we will use axiomatic approach to defining of imprecise indices which was proposed in $[1,9]$. Suppose that we used some combining rule $R$ for combining of two evidences. As a result we get new evidence. There is a question about amount of decreasing of ignorance after the using of combining rule $R$. The index of decreas- ing of ignorance will be introduced with help of imprecise indices.

There are different approaches for defining of conflict measure among belief functions. For example the well-known distance approach is considered where the conflict measure is defined with help of distance between two basic probability assignments (bpa's) [11, 12]. In this paper the measure of conflict will be introduced by axiomatically as a functional that defined on the Cartesian product of belief function sets.

We will give the basic notions and notations from Dempster-Shafer theory in section 2. The index of decreasing of ignorance will be introduced and investigated on the two bodies of evidences of special type for Dempster's and Yager's rules in section 3. The conditions will be found to guarantee the decreasing of ignorance after applying of different combining rules. The conflict measure will be axiomatically introduced and investigated in section 4 . The general view of bilinear conflict measure will be found. In section 5 the relationship between the measure of conflict and the index of decreasing of ignorance will be investigated by analytically and statistically in the case of special types of evidences and the application of Dempster rule.

\section{Theory of evidence}

\subsection{Belief function and body of evidence}

The concepts of belief and plausibility functions are the main notions of the Dempster-Shafer theory (theory of evidence). Let $X$ be a finite universal set and $2^{X}$ be the power set of $X$. Consider a belief measure (or belief function) [15] $g: 2^{X} \rightarrow[0,1]$. In the theory of evidence [14] the value $g(A), A \in 2^{X}$, is interpreted as the degree of belief that the true alternative of $X$ belongs to the set $A$ [15]. A belief function $g$ is defined in evidence theory by a set function $m_{g}(A)$, called basic probability assignment (bpa). It satisfies the following conditions [14]:

$m_{g}: 2^{X} \rightarrow[0,1], m_{g}(\varnothing)=0, \sum_{A \subseteq X} m_{g}(A)=1$.

Then $g(A)=\sum_{B: B \subseteq A} m_{g}(B)$. Let the set of all belief measures on $2^{X}$ be denoted by $\operatorname{Bel}(X)$.

Belief function $g$, and its dual, plausibility function $\bar{g}$, are considered together in evidence theory. The dual of $g$ is calculated by $\bar{g}(A)=1-g(\bar{A}), A \in 2^{X}$. 
Basic probability assignment $m_{g}$ may be computed by belief function $g$ with help of so called Möbius transform of $g: m_{g}(B)=\sum_{A: A \subseteq B}(-1)^{|B \backslash A|} g(A)$.

Belief and plausibility functions can be considered as lower and upper estimations of probabilities. Indeed, for any belief measure $g$ one can find a probability measure $p$ such that $g(A) \leq p(A) \leq \bar{g}(A)$ for all $A \in 2^{X}$. Then the pair $(g(A), \bar{g}(A))$ shows the uncertainty of probability, assigned to the event $A$.

The belief function has following statistical interpretation. Let $N$ experts were opinions (evidence) about the values of a some variable $x \in X$. Moreover $c_{i}$, $i=1, \ldots, k$, experts from $N$ assert that $x \in A_{i}$, $i=1, \ldots, k$, where $A_{i} \in 2^{X}$. We have $\sum_{i=1}^{k} c_{i}=N$. Let $m\left(A_{i}\right)=c_{i} / N$ be a frequency of evidence about $x \in A_{i}$, $i=1, \ldots, k$. The subset $A_{i} \in 2^{X}$ is called by a focal element if $m\left(A_{i}\right)>0$. Let $\mathcal{A}$ is a set of all focal elements. The set function $m(A)=m\left(A_{i}\right)$ if $A=A_{i} \in \mathcal{A}$ and $m(A)=0$ otherwise satisfies the condition (1). Then pair $F=(\mathcal{A}, m)$ is called a body of evidence. We will denote through $\mathcal{A}(g)$ and $F(g)$ the set of all focal elements and the body of evidence correspondingly related with belief function $g$.

\subsection{Combining rules in Dempster-Shafer theory}

Suppose that we have two bodies of evidence $F_{1}=\left(\mathcal{A}^{(1)}, m^{(1)}\right)$ and $F_{2}=\left(\mathcal{A}^{(2)}, m^{(2)}\right)$ which are defined on the set $X$. For example, they may be received from two different sources of information. Then we have a problem of combining of two different evidence in one evidence. The different rules of combining of evidence are considered in Dempster-Shafer theory. In general a combining rule is a some operator $R: \operatorname{Bel}(X) \times \operatorname{Bel}(X) \rightarrow \operatorname{Bel}(X)$. The more detailed and critical review of different combining rules may be found in [13]. We will mention some basic rules of combining.

a) Dempster's rule. This rule was introduced in [2] for combining of upper and lower probabilities based on the assumption that two basic probability assignments were independent. But later Shafer [14] has generalized the rule as a definition for combining of independent evidence. It rule is defined as

$$
\begin{aligned}
& m_{D}(A)=\frac{1}{1-K} \sum_{A_{1} \cap A_{2}=A} m^{(1)}\left(A_{1}\right) m^{(2)}\left(A_{2}\right), A \neq \varnothing \\
& m_{D}(\varnothing)=0, K=\sum_{A_{1} \cap A_{2}=\varnothing} m^{(1)}\left(A_{1}\right) m^{(2)}\left(A_{2}\right)
\end{aligned}
$$

The value $K$ characterizes the amount of conflict of two sources of information those described by bodies of evidence $\left(\mathcal{A}^{(1)}, m^{(1)}\right)$ and $\left(\mathcal{A}^{(2)}, m^{(2)}\right)$. If $K=1$ then sources of information are absolutely conflicting and the Dempster's rule may not be applied. This rule has subjected to numerous critics $[3,4,17-20]$. New approaches of combining of evidence were suggested as a result of this critic.

b) Discount rule. This rule was introduced by Shafer [14]. The main idea was consisted to using of some coefficient $\alpha \in[0,1]$ for discounting of basic probabilities: $\quad m^{\alpha}(A)=(1-\alpha) m(A), \quad A \neq X, \quad m^{\alpha}(X)=$ $\alpha+(1-\alpha) m(X)$. This coefficient characterizes the degree of reliability of information: if $\alpha=0$ then source of information is absolutely reliable. If $\alpha=1$ then source of information is absolutely no reliable. The Dempster's rule (2) applies after discounting. If $\alpha \in(0,1)$ then discount rule (2) may be applied for any bodies of evidence.

c) Yager's modified Dempster's rule. This rule was introduced in [19] and it is defined as

$$
\begin{gathered}
q(A)=\sum_{A_{1} \cap A_{2}=A} m^{(1)}\left(A_{1}\right) m^{(2)}\left(A_{2}\right), A \in 2^{X}, \\
m_{Y}(A)= \\
q(A), A \neq \varnothing, X, m_{Y}(\varnothing)=q(\varnothing)=K, \\
m_{Y}(X)=m_{Y}(\varnothing)+q(X),
\end{gathered}
$$

where value $K$ is defined by (2). The value $q(X)=m^{(1)}(X) m^{(2)}(X)$ characterizes the amount of ignorance in two bodies of evidence $\left(\mathcal{A}^{(1)}, m^{(1)}\right)$ and $\left(\mathcal{A}^{(2)}, m^{(2)}\right)$. Therefore Yager's rule uses information about conflict (value $q(\varnothing)$ ) and ignorance (value $q(X))$ only when computed the bpa of universal set $X$. This means that Yager's rule is very cautious rule.

d) Inagaki's unified combination rule [6]. This rule is determinated with help of set function $q(A)$ that used Yager [19] in (3) and nonnegative parameter $k$ :

$$
\begin{gathered}
m_{I}(A)=q(A)(1+k q(\varnothing)), A \neq X, \\
m_{I}(X)=q(X)(1+k q(\varnothing))+q(\varnothing)(1+k q(\varnothing)-k),
\end{gathered}
$$

where $0 \leq k \leq 1 /(1-q(\varnothing)-q(X))$. If $k=0$ then we have Yager's rule. If $k=1 /(1-q(\varnothing))$ then we get Dempster's rule. Therefore Inagaki's rule uses information about conflict and ignorance when computed the bpa of all sets with some coefficient $(1+k q(\varnothing))$ that defined relation between the conflict and ignorance.

e) Zhang's center combination rule. This rule was introduced in [21] and it is defined as

$$
m_{Z}(A)=\sum_{A_{1} \cap A_{2}=A} r\left(A_{1}, A_{2}\right) m^{(1)}\left(A_{1}\right) m^{(2)}\left(A_{2}\right), A \in 2^{X},
$$

where $r\left(A_{1}, A_{2}\right)$ be a measure of intersection of sets $A_{1}$ and $A_{2}$. For example $r\left(A_{1}, A_{2}\right)=c\left|A_{1} \cap A_{2}\right| /\left|A_{1}\right|\left|A_{2}\right|$ or 
$r\left(A_{1}, A_{2}\right)=c\left|A_{1} \cap A_{2}\right| /\left|A_{1} \cup A\right|$ Jaccard similarity coefficient, where $c>0$ is a normalizing factor.

f) Dubois and Prade's disjunctive consensus rule. This rule was introduced in [5] and it is defined as

$m_{D P}(A)=\sum_{A_{1} \cup A_{2}=A} m^{(1)}\left(A_{1}\right) m^{(2)}\left(A_{2}\right), A \in 2^{X}$.

There are more other combination rules. These examples of combination rules show us that we must take into account the information about conflict and ignorance when we make combination of evidence. Below we define the functionals by which we will calculate the quantity of conflict and ignorance in every concrete situation of combining.

\section{Changing of ignorance after application of com- bining rules}

The effectiveness of applying of combining rule may be estimated by quantity of decreasing of ignorance after the using of combining rule. We will use the notion of imprecision index for calculate the quantity of ignorance. In general imprecision index $f$ is defined on the set $\operatorname{Bel}(X)$ and it characterizes the degree of deviation (measure of uncertainty) of belief function $g$ from probability measure. We show that this measure of uncertainty may be considered as a level of information ignorance contained in the measure $g$. We want the value of ignorance would decrease after using of combining rule. The degree of such decreasing may be estimated with help of comparison $f(g)$ with $f\left(g_{1}\right)$ and $f\left(g_{2}\right)$ where the $g$ be a belief function after a combination of evidence $F\left(g_{1}\right)$ and $F\left(g_{2}\right)$.

\subsection{Imprecise indices}

Measuring uncertainty plays a major role in uncertainty theories, in particular, probability theory, information theory, fuzzy sets theory, theory of evidence and so on. There are some ways how to define such measures in the theory of evidence. We will follow approach that was introduced in $[1,9]$.

Let we know only that the "true" alternative is in a nonempty set $B \subseteq X$. This situation can be described by the non-additive measure (the so-called primitive belief function)

$$
\eta_{\langle B\rangle}(A)=\left\{\begin{array}{l}
1, B \subseteq A \\
0, B \varsubsetneqq A
\end{array}, A \subseteq X, B \neq \varnothing\right.
$$

which gives the lower probability of an event $A$, and Hartley's measure $H\left(\eta_{\langle B\rangle}\right)=\log _{2}|B|$ can be justified. It is easily seen that nature of uncertainty associates with imprecision of the information. Hartley's measure characterizes the degree of imprecision of the information about belonging of "true" alternative.
The generalization of this case consists in the following. Let $g \in \operatorname{Bel}(X)$. Consider a pair $(g, \bar{g})$, $g(A) \leq \bar{g}(A)$ for all $A \in 2^{X}, g(\varnothing)=\bar{g}(\varnothing)=0$. We believe that there is a "true" probability measure $P$ on $2^{X}$ with $g(A) \leq P(A) \leq \bar{g}(A)$ for all $A \in 2^{X}$. In other words, set functions $g, \bar{g}$ give us upper and lower bounds of probabilities, and for any event $A \in 2^{X}$ we have only the interval $[g(A), \bar{g}(A)]$ of possible values of a "true" probability $P(A)$.

They are generalized Hartley's measure. Let $g$ be a belief function, i.e. it can be represented by $g=\sum_{B \in 2^{x}} m(B) \eta_{\langle B\rangle}$, where $m(\varnothing)=0, m(B) \geq 0$ for all $B \in 2^{X}$ and $\sum_{B \in 2^{X}} m(B)=1$. Then generalized Hartley's measure is defined by

$$
G H(g)=\sum_{B \in 2^{X} \backslash\{\varnothing\}} m(B) \log _{2}|B| .
$$

Definition 1. A functional $f: \operatorname{Bel}(X) \rightarrow[0,1]$ is called imprecision index if the following conditions are fulfilled: 1) if $g$ be a probability measure then $f(g)=0 ; 2) \quad f\left(g_{1}\right) \geq f\left(g_{2}\right)$ for all $g_{1}, g_{2} \in \operatorname{Bel}(X)$ such that $\left.g_{1} \leq g_{2} ; 3\right) f\left(\eta_{\langle X\rangle}\right)=1$.

An imprecision index $f$ on $\operatorname{Bel}(X)$ is called linear if for any linear combination $\sum_{j=1}^{k} \alpha_{j} g_{j} \in \operatorname{Bel}(X)$, $\alpha_{j} \in \mathbb{R}, \quad g_{j} \in \operatorname{Bel}(X), \quad j=1, \ldots, k, \quad$ we have $f\left(\sum_{j=1}^{k} \alpha_{j} g_{j}\right)=\sum_{j=1}^{k} \alpha_{j} f\left(g_{j}\right)$.

We notice first that belief function $g$ may be represents as a linear combination of primitive belief functions $\eta_{\langle B\rangle}$ :

$$
g=\sum_{B \in 2^{X} \backslash\{\varnothing\}} m_{g}(B) \eta_{\langle B\rangle}
$$

The set of $\left\{\eta_{\langle B\rangle}\right\}, B \in 2^{X} \backslash\{\varnothing\}$, is a basis in the set $\operatorname{Bel}(X)$ in the sense that any belief function $g \in \operatorname{Bel}(X)$ is represented uniquely as a convex combination of primitive measures $\left\{\eta_{\langle B\rangle}\right\}, B \in 2^{X} \backslash\{\varnothing\}$. On the other hand any linear functional $f$ on $\operatorname{Bel}(X)$ is defined uniquely by its values on a chosen basis of $\operatorname{Bel}(X)$. This enables to define $f$ by the set function $\mu_{f}: 2^{X} \rightarrow \mathbb{R} \quad$ with the following property $\mu_{f}(B)=f\left(\eta_{\langle B\rangle}\right), B \in 2^{X} \backslash\{\varnothing\}$. We take by definition that $\mu_{f}(\varnothing)=0$ for any linear imprecision index $f$. The different representations of imprecision index were found in [9]. In this article we will use the simplest representation that is received from Definition 1 and formula (5) directly.

Proposition 1. The functional $f: \operatorname{Bel}(X) \rightarrow[0,1]$ is a linear imprecision index on $\operatorname{Bel}(X)$ iff 


$$
f(g)=\sum_{B \in 2^{X} \backslash\{\varnothing\}} m_{g}(B) \mu_{f}(B),
$$

where set function $\mu_{f}$ satisfies the conditions:

1) $\mu_{f}(\{x\})=0$ for any $x \in X$;

2) $\mu_{f}(X)=f\left(\eta_{\langle X\rangle}\right)=1$;

3) $\mu_{f}$ be a monotonic set function i.e. $\mu_{f}\left(B^{\prime}\right) \leq \mu_{f}\left(B^{\prime \prime}\right)$ if $B^{\prime} \subseteq B^{\prime \prime}$.

The generalized normalized Hartley's measure $G H_{0}=G H / \log _{2}|X|$ (see formula (1)) is an example of linear imprecision index. A formula (4) and (6) shows us that linear imprecision index $f$ determines some distribution on the body of evidence. This distribution has a some density $\mu_{f}$. The greater value of density corresponds to the focal element which is greater by cardinality. The availability of great by cardinality and weight evidence characterizes the greater degree of ignorance. Therefore the value of linear imprecision index $f(g)$ estimates this degree of ignorance.

Notice that the set of all linear imprecision indices is a convex set.

\subsection{Index of decreasing of ignorance}

Suppose that we have two bodies of evidence $F\left(g_{1}\right)=\left(\mathcal{A}^{(1)}, m^{(1)}\right)$ and $F\left(g_{2}\right)=\left(\mathcal{A}^{(2)}, m^{(2)}\right)$ which are defined on the set $X$. These bodies of evidence corresponds belief functions $g_{1}$ and $g_{2}$ correspondingly. Let $f: \operatorname{Bel}(X) \rightarrow[0,1]$ be a some linear imprecision index that estimates the degree of ignorance contained in the measure $g$. Suppose that we used some combining rule $R$ for combining of evidence $F\left(g_{1}\right)$ and $F\left(g_{2}\right)$. As a result we get new belief function $g=R\left(g_{1}, g_{2}\right)$. Then we have a question about amount of decreasing of ignorance after the using of combining rule $R$. The degree of such decreasing may be estimated with help of comparison $f(g)$ with $f\left(g_{1}\right)$ and $f\left(g_{2}\right)$. For example we may introduce the following indices of decreasing of ignorance

$$
\begin{gathered}
I_{R}\left(g_{i} \mid g_{j}\right)=f\left(g_{i}\right)-f\left(R\left(g_{i}, g_{j}\right)\right), i, j \in\{1,2\}, \\
I_{R}\left(g_{1}, g_{2}\right)=\min \left\{I_{R}\left(g_{1} \mid g_{2}\right), I_{R}\left(g_{2} \mid g_{1}\right)\right\} .
\end{gathered}
$$

The decreasing of ignorance corresponded to the case of positivity of index $I_{R}\left(g_{1}, g_{2}\right)$. Notice these indices were introduced in [8].

We consider some partial cases of variation of ignorance when evidences are combined. The following situation of "consensual evidences" is a very important case of combining. Let $\mathcal{A}^{(1)}$ and $\mathcal{A}^{(2)}$ are the two sets of focal elements satisfying the conditions:

1) $A^{\prime} \cap A^{\prime \prime}=\varnothing, B^{\prime} \cap B^{\prime \prime}=\varnothing$ for all $A^{\prime}, A^{\prime \prime} \in \mathcal{A}^{(1)}$, $B^{\prime}, B^{\prime \prime} \in \mathcal{A}^{(2)}$;

2) for every $A \in \mathcal{A}^{(1)}$ exists a unique $B \in \mathcal{A}^{(2)}$ such that $A \cap B \neq \varnothing$;
3) for every $B \in \mathcal{A}^{(2)}$ exists a unique $A \in \mathcal{A}^{(1)}$ such that $A \cap B \neq \varnothing$.

Thus there is a one-to-one correspondence $\varphi$ between the elements of sets $\mathcal{A}^{(1)}$ and $\mathcal{A}^{(2)}$.

Consensual evidences appear, for example, in cases where two experts give us the information in the some "outlined framework" (eg, optimistic and pessimistic scenarios for the development of a situation).

If two sets of focal elements satisfy the conditions 1)$3)$ and the additional condition

4) $A \subseteq \varphi(A)$ for all $A \in \mathcal{A}^{(1)}$ then we will call this situation by "clarifying evidences".

We have a question about decreasing of ignorance after combining of "consensual evidences". It turns out that the answer on this question depends from combining rule. At first we formulate the result about combining of consensual evidences with help of Dempster's rule. Then the following statement about the index of decreasing of ignorance is true.

Proposition 2. Let $F\left(g_{1}\right)=\left(\mathcal{A}^{(1)}, m^{(1)}\right) \quad$ and $F\left(g_{2}\right)=\left(\mathcal{A}^{(2)}, m^{(2)}\right)$ are the two bodies of evidence satisfying the conditions 1$)-3)$. Then the index of decreasing of ignorance $I_{D}\left(g_{1}, g_{2}\right)$ will positive for Dempster's rule if the following condition is true:

$$
\begin{gathered}
\sum_{A \in \mathcal{A}^{(1)}} m^{(1)}(A) m^{(2)}(\varphi(A))> \\
\max _{A \in \mathcal{A}^{(1)}} \mu_{f}(A \cap \varphi(A)) \max \left\{\frac{m^{(1)}(A)}{\mu_{f}(\varphi(A))}, \frac{m^{(2)}(\varphi(A))}{\mu_{f}(A)}\right\}
\end{gathered}
$$

The condition (7) is simplified in the case of "clarifying evidences".

Corollary 1. Let two bodies of evidence $F\left(g_{1}\right)=\left(\mathcal{A}^{(1)}, m^{(1)}\right)$ and $F\left(g_{2}\right)=\left(\mathcal{A}^{(2)}, m^{(2)}\right)$ satisfy the conditions 1)-4). Then the index of decreasing of ignorance $I_{D}\left(g_{1}, g_{2}\right)$ will positive for Dempster's rule if the following condition is true:

$$
\begin{gathered}
\sum_{A \in \mathcal{A}^{(1)}} m^{(1)}(A) m^{(2)}(\varphi(A))> \\
\max _{A \in \mathcal{A}^{(1)}} \max \left\{m^{(1)}(A) \frac{\mu_{f}(A)}{\mu_{f}(\varphi(A))}, m^{(2)}(\varphi(A))\right\} .
\end{gathered}
$$

The expression on the left in (7) (or $\left(7^{\prime}\right)$ ) is a scalar product two vector-evidences (or correlation between the two evidences). Therefore this expression has great value if vector-evidences are collinear (or consensual). Thus the inequality (7) (or ( $\left.7^{\prime}\right)$ ) means that great correlation of evidences is a sufficient condition for decreasing of ignorance after applying combining rule.

We have following proposition for Yager's rule.

Proposition 3. Let $F\left(g_{1}\right)=\left(\mathcal{A}^{(1)}, m^{(1)}\right) \quad$ and $F\left(g_{2}\right)=\left(\mathcal{A}^{(2)}, m^{(2)}\right)$ are the two bodies of evidence satisfying the conditions 1)-3). Then the index of decreasing of ignorance $I_{Y}\left(g_{1}, g_{2}\right)$ will positive for Yager's rule iff 


$$
\begin{gathered}
\sum_{A \in \mathcal{A}^{(1)}} m^{(1)}(A) m^{(2)}(\varphi(A))\left(1-\mu_{f}(A \cap \varphi(A))\right)> \\
\max \left\{\sum_{A \in \mathcal{A}^{(1)}} m^{(1)}(A)\left(1-\mu_{f}(A)\right), \quad \sum_{A \in \mathcal{A}^{1)}} m^{(2)}(\varphi(A))\left(1-\mu_{f}(\varphi(A))\right)\right\} .
\end{gathered}
$$

But the ignorance can't be decreased for clarifying evidences with help of Yager's rule.

Corollary 2. Let two bodies of evidence $F\left(g_{1}\right)=\left(\mathcal{A}^{(1)}, m^{(1)}\right)$ and $F\left(g_{2}\right)=\left(\mathcal{A}^{(2)}, m^{(2)}\right)$ satisfy the conditions 1)-4). Then the index of decreasing of ignorance $I_{Y}\left(g_{1} \mid g_{2}\right)=f\left(g_{1}\right)-f\left(Y\left(g_{1}, g_{2}\right)\right) \quad$ will nonpositive for Yager's rule.

\section{Conflict measure}

The measure of conflict will be introduced and will research in this section. The many works are devoted to analyzing of combinations of conflicting belief functions in framework of Dempster-Shafer theory. The value $K$ is used as a measure of conflict in most researches. But in sometimes the large value of $K$ does not means the availability of large conflict between the evidence (see [10]). Therefore the other measures of conflict were introduced in a number of works [7, 10, 16].

Below we introduce the measure of conflict axiomatically with help of some measure of intersection of sets.

Let $\quad F_{1}=\left(\mathcal{A}^{(1)}, m^{(1)}\right), \quad \mathcal{A}^{(1)}=\mathcal{A}\left(g_{1}\right) \quad$ and $F_{2}=\left(\mathcal{A}^{(2)}, m^{(2)}\right), \mathcal{A}^{(2)}=\mathcal{A}\left(g_{2}\right)$ are the two bodies of evidence on $X$ related with belief functions $g_{1}$ and $g_{2}$ correspondingly. We will introduce the notion of measure of conflict of bodies of evidence $F_{1}$ and $F_{2}$.

Let $r: 2^{X} \times 2^{X} \rightarrow[0,1]$ be some measure of intersection of sets (see the Zhang's center combination rule) that satisfy following conditions:
1) $r(A, B)=r(B, A)$
2) $r(A, B)=0$ if $A \cap B=\varnothing$;
3) $r(A, A)=1, A \neq \varnothing$.

Definition 2. A functional $c_{r}: \operatorname{Bel}(X) \times \operatorname{Bel}(X) \rightarrow[0,1]$ is called by measure of conflict with respect to measure of intersection $r$ if the following condition are fulfilled:

1) $c_{r}\left(g_{1}, g_{2}\right)=c_{r}\left(g_{2}, g_{1}\right)$ for all $g_{1}, g_{2} \in \operatorname{Bel}(X)$;

2) $c_{r}\left(g^{\prime}, g\right) \geq c_{r}\left(g^{\prime \prime}, g\right)$ if $F\left(g^{\prime}\right)=F \cup\left(A^{\prime}, m\right)$,

$F\left(g^{\prime \prime}\right)=F \cup\left(A^{\prime \prime}, m\right)$ and $r\left(A^{\prime}, B\right) \leq r\left(A^{\prime \prime}, B\right)$ for all $B \in \mathcal{A}(g)$;

3) $c_{r}\left(g_{1}, g_{2}\right)=1$ if $A \cap B=\varnothing$ for all $A \in \mathcal{A}\left(g_{1}\right)$, $B \in \mathcal{A}\left(g_{2}\right)$.

A measure of conflict $c_{r}$ on $\operatorname{Bel}(X) \times \operatorname{Bel}(X)$ is called bilinear if $c_{r}\left(\alpha g_{1}+\beta g_{2}, g\right)=$ $\alpha c_{r}\left(g_{1}, g\right)+\beta c_{r}\left(g_{2}, g\right)$ for all $\alpha, \beta \in[0,1], \alpha+\beta=1$, $g, g_{1}, g_{2} \in \operatorname{Bel}(X)$.

Proposition 4. A functional $c_{r}$ be a bilinear measure of conflict on $\operatorname{Bel}(X) \times \operatorname{Bel}(X)$ iff

$$
\begin{gathered}
c_{r}\left(g_{1}, g_{2}\right)=\sum_{A \in \mathcal{A}\left(g_{1}\right), B \in \mathcal{A}\left(g_{2}\right)} \gamma(A, B) m^{(1)}(A) m^{(2)}(B)= \\
K+\sum_{A \cap B \neq \varnothing} \gamma(A, B) m^{(1)}(A) m^{(2)}(B),
\end{gathered}
$$

where $\gamma(A, B) \in[0,1]$ satisfy following conditions:

a) $\gamma(A, B)=\gamma(B, A)$

b) $\gamma\left(A^{\prime}, B\right) \geq \gamma\left(A^{\prime \prime}, B\right)$ if $r\left(A^{\prime}, B\right) \leq r\left(A^{\prime \prime}, B\right)$;

c) $\gamma(A, B)=1$ if $A \cap B=\varnothing$.

For example, the measure of conflict $\gamma(A, B)=c_{r}\left(\eta_{\langle A\rangle}, \eta_{\langle B\rangle}\right)=\varphi(r(A, B)), \quad A, B \neq \varnothing$, satisfy the conditions a)-c) of Proposition 4 if $\varphi$ is a nonincreasing function for which $\varphi(1)=0, \varphi(0)=1$ and $r(A, B)$ satisfies the conditions 1$)-3)$.

In this case $c_{r}\left(g_{1}, g_{2}\right)=K$ if

$$
r(A, B)=\left\{\begin{array}{l}
1, A \cap B \neq \varnothing, \\
0, A \cap B=\varnothing
\end{array}\right.
$$

be a primitive measure of intersection.

The measure of conflict may be used for preliminary estimating quantity of conflict for two evidences.

Example. Let $F\left(g_{1}\right)=\left(\mathcal{A}^{(1)}, m^{(1)}\right) \quad$ and $F\left(g_{2}\right)=\left(\mathcal{A}^{(2)}, m^{(2)}\right)$ are two bodies of consensual evidences (see 3.2). Then

$c_{r}\left(g_{1}, g_{2}\right)=1-\sum_{A \in \mathcal{A}^{(1)}}(1-\gamma(A, \varphi(A))) m^{(1)}(A) m^{(2)}(\varphi(A))$.

In particular if

$$
\gamma(A, B)=1-r(A, B)=|A \cap B| / \min \{|A|,|B|\}
$$

then

$c_{r}\left(g_{1}, g_{2}\right)=1-\sum_{A \in \mathcal{A}^{(1)}} \frac{|A \cap \varphi(A)|}{\min \{|A|,|\varphi(A)|\}} m^{(1)}(A) m^{(2)}(\varphi(A))$.

If we have two bodies of clarifying evidences then the last expression is simplified as

$$
c_{r}\left(g_{1}, g_{2}\right)=K=1-\sum_{A \in \mathcal{A}^{(1)}} m^{(1)}(A) m^{(2)}(\varphi(A)) \text {. }
$$

\section{Studying the relation between measure of conflict and index of decreasing of ignorance}

We investigate the relationship between the measure of conflict and the index of decreasing of ignorance in the case of different types of evidences and the application of different combining rules. 


\subsection{Statistical investigation}

We will carry out a uniform generation of focal elements of a particular type of evidence and the basic probability assignments. We restrict by generation of bodies of evidence which have capacity is equal two. Some results of statistical analysis are given below for the two body types of combinable evidence.

\subsubsection{General case of combinable evidence}

Firstly we consider the general case of combining of evidence.

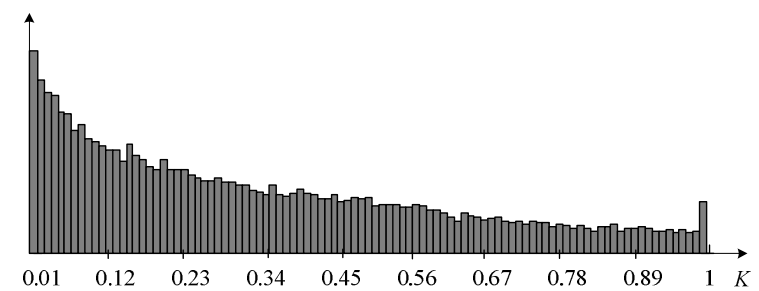

Fig. 1: The histogram of conflict measure in general case.

The histogram of conflict measure in general case (see Fig. 1) shows us that the number of evidence with great conflict is less than the number of evidence with small conflict.

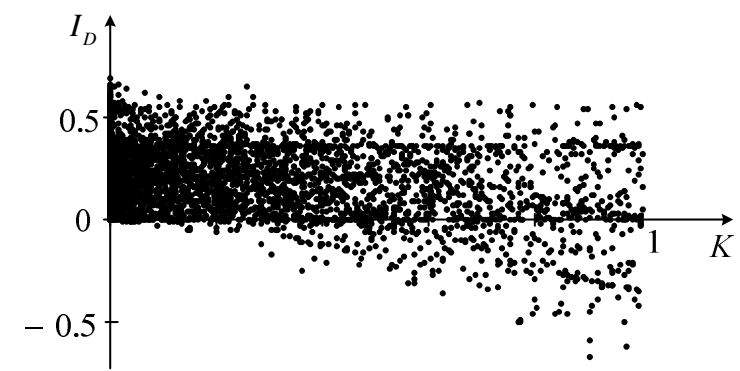

Fig. 2: Distribution of points $\left(K, I_{D}\right)$ when combining the two evidence in general case.

The mean value of the index of decreasing of ignorance decreases with increasing values of the conflict measure, but remains positive (see Fig. 2). The value of variance increases weakly.

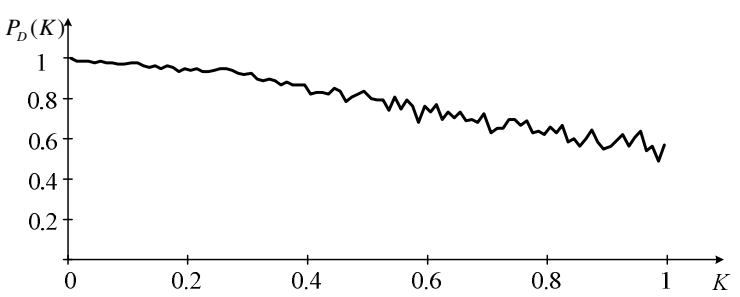

Fig. 3: The dependence of the estimate of the probability

$P_{D}(K)=P\left\{I_{D}>0 \mid K\right\}$ from conflict in general case.

The Fig. 3 shows us that the probability of nonnegativity of index of decreasing of ignorance is greater than 0.5 for all values of the measures of conflict in general case of combining of evidence.

\subsubsection{Clarifying evidence}

Now we consider the case of clarifying evidence. In this case the distribution of the conflict measure and the relationship between the conflict measure and the index of decrease of ignorance will be another.

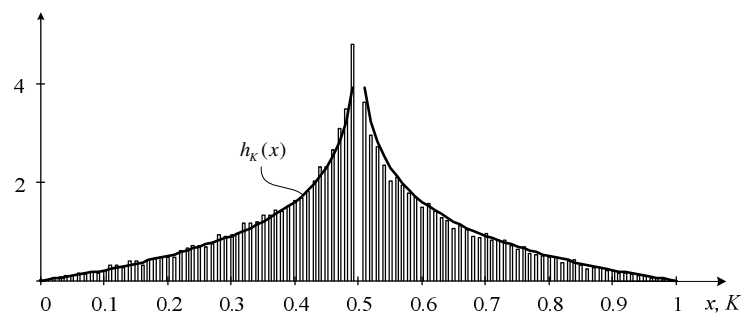

Fig. 4: The histogram and function of density of conflict measure in case of clarifying evidences.

The Fig. 4 shows us that the evidences with median conflict are a most common the clarifying evidence.

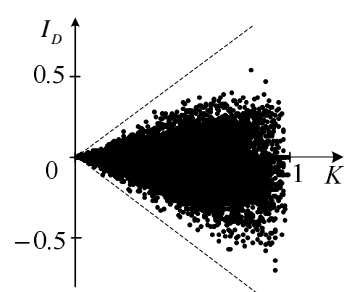

Fig. 5: Distribution of points $\left(K, I_{D}\right)$ when combining the two clarifying evidences.

The mean value of the index of decreasing of ignorance decreases with increasing values of the conflict measure and it will be negative in general (see Fig. 5). The value of variance increases strongly.

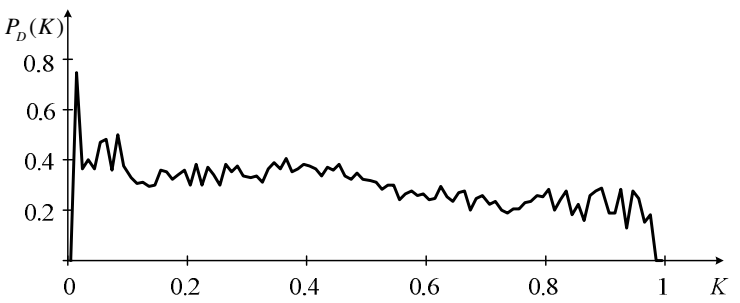

Fig. 6: The dependence of the estimate of the probability $P_{D}(K)=P\left\{I_{D}>0 \mid K\right\}$ from conflict in case of clarifying evidences.

The Fig. 6 shows us that the probability of nonnegativity of index of decreasing of ignorance is less than 0.5 for almost all values of the conflict measure in case of clarifying evidences.

\subsection{Analytical investigation}

\subsubsection{Upper and lower estimates of the relationship} between the conflict measure and index of decreasing of ignorance

We now consider the index of decreasing of ignorance in case of combining the evidence by the rule $R$, provided that the measure of conflict $c\left(g_{1}, g_{2}\right)=K$. Then the values 


$$
\begin{gathered}
\bar{I}_{R}(K)=\sup \left\{I_{R}\left(g_{1}, g_{2}\right): c\left(g_{1}, g_{2}\right)=K\right\}, \\
\underline{I}_{R}(K)=\inf \left\{I_{R}\left(g_{1}, g_{2}\right): c\left(g_{1}, g_{2}\right)=K\right\}
\end{gathered}
$$

characterize the upper and lower estimates of the relationship between the conflict measure and index of decreasing of ignorance.

The following statement is true for the values $\bar{I}_{D}(K)$ and $\underline{I}_{D}(K)$ in the case of a combination of clarifying evidence.

Proposition 5. Let we combine two clarifying evidence of cardinality 2 , the conflict measure computed from formula (2) and the index of decreasing of ignorance computed with help of generalized normalized Hartley's measure. Then we have $\bar{I}_{D}(K) \leq K$, $\underline{I}_{D}(K) \geq-K$.

Graphics of upper and lower bounds of functions $\bar{I}_{D}(K)$ and $\underline{I}_{D}(K)$ correspondingly are indicated by dashed in Fig. 5.

\subsubsection{Distribution of random conflict measure}

We now find the theoretical distribution of the conflict measure in case of clarifying evidences provided that the basic probability assignments of these evidences have a uniform distributions.

Proposition 6. Let two clarifying bodies of evidence have a cardinality which equal two and their basic probability assignments have uniform distributions. Then the random variable - conflict measure $K$, which is calculated by the formula (2), has a probability density equal to $h_{K}(x)=-\ln |2 x-1|, x \in[0,1 / 2) \cup(1 / 2,1]$.

Graphics of function $h_{K}(x)$ is indicated by solid in Fig. 4.

\section{Summary and conclusion}

There is a problem of choose combining rule at the Dempster-Shafer theory. This problem does not have a complete solution. It connected with uncertainty of description of evidence in frame of Dempster-Shafer theory. The correctness of the combination may depend on context of information but not only on structure of information. However in the some cases we can get the information about quality of combining. The solution of this problem is associated with analyses of quantity of ignorance and conflict of evidence. At this article were introduced the index of decreasing of ignorance and measure of conflict for calculating of ignorance and conflict of evidence. These measures are investigated for some important cases of evidence and different combining rules. It is shown that the great correlation between the bodies of evidence is a sufficient condition of decreasing of ignorance after the applying of combining rule. If we have many bodies of evidence (for example, we have many sources of information) then we may used defined measures for optimal choice of bodies of evidence for combining.

\section{Acknowledgements}

The study was implemented in the framework of The Basic Research Program of the Higher School of Economics. This work was supported by the grant 11-0700591 of RFBR (Russian Foundation for Basic Research).

\section{References}

[1] A. Bronevich and A. Lepskiy, An axiomatic approach to the definition of imprecision index of fuzzy measures. In Proceedings of the $2^{\text {nd }}$ International scientific seminar "Integrative models and soft computing in artificial intelligence", Science Press of mathematical literature, Kolomna, pages 127-130, 2003. (in Russian)

[2] A.P. Dempster, Upper and Lower Probabilities Induced by a Multivalued Mapping, The Annals of Statistics 28:325--339, 1967.

[3] H. Deqiang, H. Chongzhao and Y. Yi, A modified evidence combination approach based on ambiguity measure. In Proceedings of the 11th International Conference on Information fusion, pages 1-6, 2008.

[4] D. Dubois and H. Prade, Representation and combination of uncertainty with belief functions and possibility measures, Computational Intelligence 4:244--264, 1988.

[5] D. Dubois and H. Prade, On the combination of evidence in various mathematical frameworks, Reliability Data Collection and Analysis. J. Flamm and T. Luisi. Brussels, ECSC, EEC, EAFC, pages 213241, 1992.

[6] T. Inagaki, Interdependence between SafetyControl Policy and Multiple-Sensor Schemes Via Dempster-Shafer Theory, IEEE Transactions on Reliability 40(2):182--188, 1991.

[7] E. Lefevre, O. Colot and P. Vannoorenberghe, Belief function combination and conflict management, Information Fusion 3:149--162, 2002.

[8] A. Lepskiy, Estimation of Conflict and Decreasing of Ignorance in Dempster-Shafer Theory, Procedia Computer Science 17:1113--1120, 2013.

[9] A. Lepskiy and A. Bronevich, Various representations and algebraic structure of linear imprecision indices. In Proceedings of the $5^{\text {th }}$ EUSFLAT Conference, Ostrava, Chezh Republic, September 1114, 1:297--304, 2007.

[10] W. Liu, Analyzing the degree of conflict among belief functions, Artificial Intelligence 170(11): 909--924, 2006.

[11] Z. Liu, J. Dezert and Q. Pan, A new measure of dissimilarity between two basic belief assignments, hal-00488045, version 1 - 1 Jun 2010.

[12] A. Martin, A.-L. Jousselme and C. Osswald, Conflict measure for the discounting operation on belief functions. In Proceedings of the 11th International Conference on Information fusion, pages 1-8, 2008.

[13] K. Sentz and S. Ferson, Combination of evidence in Dempster-Shafer theory, Report SAND 2002-0835, Sandia National Laboratories, 2002. 
[14] G. Shafer, A Mathematical Theory of Evidence, Princeton, N.J.: Princeton University Press, 1976.

[15] P. Smets, Belief functions and transferable belief model, http://ippserv.rug.ac.be.

[16] P. Smets, Analyzing the combination of conflicting belief functions, Information Fusion 8(4):387--412, 2007.

[17] Q. Sun, X. Q. Ye and W.K. Gu, A new combination rules of evidence theory, Acta Electronica Sinica 28(8):117--119, 2000.

[18] G. Xin, Y. Xiao and H. You, An Improved Dempster-Shafer Algorithm for Resolving the Conflicting Evidences, International Journal of Information Technology, 11(12):68--75, 2005.
[19] R. Yager, On the Dempster-Shafer Framework and New Combination Rules, Information Sciences 41:93--137, 1987.

[20] L.A. Zadeh, Review of Books: A Mathematical Theory of Evidence, The AI Magazine 5(3):81-83, 1984.

[21] L.A. Zhang, Representation, independence, and combination of evidence in the DempsterShafer theory, Advances in the Dempster-Shafer Theory of Evidence. R.R. Yager, J. Kacprzyk and M. Fedrizzi. New York, John Wiley \& Sons, Inc. pages 51-69, 1994. 